\title{
Diagnóstico de bullying en escuelas focalizadas del municipio de Santa Ana
}

\author{
Miguel Ángel Pleitez Herrera \\ Maestro en Asesoría Educativa \\ Investigador \\ Marca de Servicios Culturales Cuiloa Nu Tal, El Salvador \\ pleitezdante26@yahoo.com
}

Recepción: 08-08-2018 / Aceptación: 10-11-2018

\section{Resumen}

Este artículo presenta un diagnóstico realizado en una muestra de las escuelas focalizadas del municipio de Santa Ana como un insumo para la Unidad de Arte, Cultura, Recreación, Deportes y Ciudadanía del Ministerio de Educación, Ciencia y Tecnología (MINEDUCYT).

La investigación descriptiva abordo nueve de treinta escuelas focalizadas, haciendo un total de 117 sujetos, entre estos: directivos, docentes y estudiante. El bullying no es una situación nueva; en la mayoría de los casos, se asume su existencia. Pero, lastimosamente, se carece de un cuerpo teórico-práctico que indique la forma en que este se desarrolla, a fin de poder intervenirlo y paulatinamente erradicarlo. Por esa razón, se describen las manifestaciones de este flagelo y determinan cuáles son los mecanismos vertidos en el Manual de Convivencia Escolar para su tratamiento.

Este proceso, por su carácter sensible y socioeducativo, permitió encontrar manifestaciones diversas: por un lado, el temor de los directivos por considerar el fenómeno como una falencia administrativa, más que un problema social que debe ser intervenido de forma adecuada, en beneficio de toda la comunidad educativa; y, por otro lado, optimismo de parte de docentes y estudiantes, ya que se hizo visible una problemática que afecta a todos. Todo lo anterior contribuyó a la construcción de un recurso investigativo que permitirá el planteamiento de futuras acciones para intervenir el bullying de forma pronta, precisa y adecuada.

Palabras clave: Bullying, convivencia escolar, escuelas focalizadas, procedimientos para la resolución de conflictos.

\begin{abstract}
This article shows a diagnosis about selected schools in Santa Ana City as an input for the Unidad de Arte, Cultura, Recreación, Deportes y Ciudadanía del Ministerio de Educación, Ciencia y Tecnología (MINEDUCYT).

The descriptive research addressed nine of thirty selected schools, making 117 subjects including principals, teachers, and student. Bullying is not a new situation; in most cases, its existence is assumed. Unfortunately, it lacks a theoretical-practical structure that indicates the way in which it develops in order to intervene and gradually eradicate it. For this reason, the manifestations of this scourge are described in order to determine which mechanisms are poured into the School Coexistence Manual for its treatment.

This process, due to its sensitive and socio-educational nature, made it possible to find different manifestations, on one hand, the principles' fear of considering the phenomenon as an administrative failure rather than a social problem that must be faced with appropriate ways in order to benefit the entire educational community. On the other hand, teachers and students showed optimisms since a problem that affects everyone is becoming more and more visible.
\end{abstract}

All of the aforementioned contributed to create a research resource that will allow the approach of future actions to intervene bullying in a prompt, precise, and adequate way.

Keywords: Bullying, school coexistence, selected schools, procedures for conflict resolution. 


\section{Introducción}

Si las relaciones de convivencia dentro de las escuelas son efectivas y de común acuerdo, todos participan con libertad de opinar. Sin duda, el clima educativo será positivo y permitirá el crecimiento de todos sus miembros, pero si estas no se dan de tal manera y se fundamentan en situaciones de violencia, el producto obtenido será nocivo para todos los que ahí convergen.

Partiendo de lo anterior, el gestar iniciativas que den pauta de cómo se desarrolla la convivencia escolar son de mucho beneficio para el espectro educativo; pues abren la posibilidad de construir mecanismos concretos que permitan mermar ciertas acciones que estén afectando a la comunidad.

Este diagnóstico se realizó en realidades educativas, consideradas por el Ministerio de Educación, Ciencia y Tecnología (MINEDUCYT), como sensibles o de riesgo social; por esa razón, pertenecen a una categoría denominada: "Atención técnica focalizada". Este tipo de asistencia se orienta a lo que establecen Fiabane, Yáñez y Campos (2009) como

[...] cada escuela [...] es diferente a las demás. Las razones de perturbaciones son variadas, y las escuelas tienen distintas formas de reaccionar frente a esta situación. Por lo tanto, no existe una solución mágica para sus dificultades. Necesitan diferentes tipos de ayuda. (p.18)
Las dificultades focalizadas en este marco son de tipo social; a este respecto en el año 2013, la Comisión Interinstitucional e Intergremial del Sector docente coordinada por el MINEDUCYT $^{1}$, presentó el "Plan de Prevención y Seguridad Escolar", que busca articular a todos los actores responsables de la prevención de la violencia escolar, para impulsar acciones que contribuyan a mejorar la seguridad y cultura de convivencia en el entorno educativo (MINEDUCYT, 2013).

En esas circuntancias, se centró la atención en un fenómeno que afecta de forma negativa la relaciones entre los estudiantes en la comunidad educativa. En español se le conoce como "acoso entre pares" u "hostigamiento escolar"; pero dentro del contexto de la investigción se manejará el termino en inglés, propuesto aproximadamente en el año 1973 en Noruega, el cual es: bullying.

Según lo que establece Díaz (2009), el primer trabajo sistemático sobre la conducta bullying fue realizado por Dan Olweus, profesor de la Universidad de Bergen. Se trata de un estudio longitudinal que comenzó en 1970, y aún hoy sigue desarrollándose. Aunque este término no es nuevo, sus afectaciones son actuales y están flagelando el normal desarrollo de la convivencia escolar.

En el caso de El Salvador, los estudios existentes respecto a la temática han sido ejecutados por instancias diferentes del MINEDUCYT. Ante eso, una de las necesidades latentes fue

1. Dentro del documento, el autor se referirá al término mediante este acrónimo. 
formular un insumo investigativo que diera líneas de acción a nivel de Sistema Educativo Nacional; siendo esta investigación la que planteó ese precedente, dando un marco teórico-práctico real sobre las manifestaciones del bullying en escuelas del municipio de Santa Ana, pasando de un punto de vista especulativo a uno académico en concreto.

Cerezo (1991) citado en (ALBOR-COHS, 2000) define bullying como

[...] la violencia mantenida, mental o física, guiada por un escolar o por un grupo, dirigida contra otro escolar que no es capaz de defenderse a sí mismo. Puede adoptar diversas formas, unas más directas como la agresión física y verbal, y otras encubiertas o indirectas como la exclusión social. (p.10)

Es importante destacar, que para que se considere bullying debe ser una conducta guiada y repitente hacia un mismo estudiante; sino existe esa premisa, solamente es una conducta de violencia aislada, pues no tiene una durabilidad en tiempo y sujeto. En relación con todo lo planteado, este fenómeno tiene una tipología específica que denota qué situaciones pueden ser consideras así. Al respecto, en la figura 1 se muestra los diferentes escenarios donde puede manifestarse.

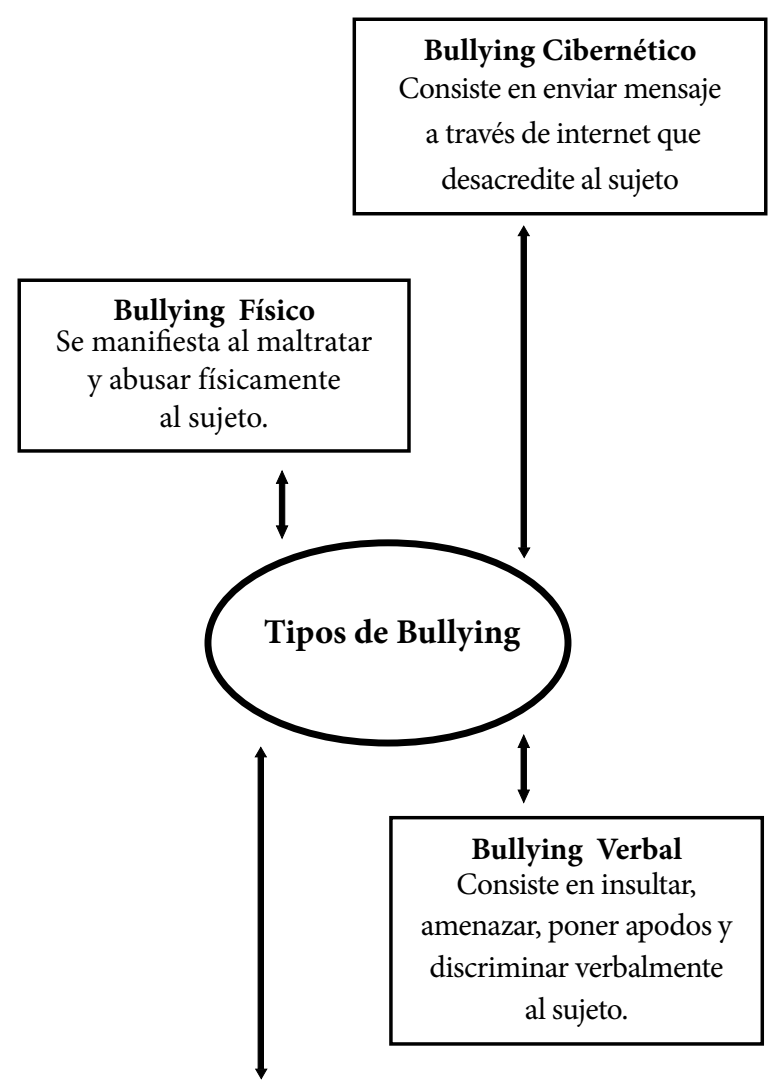

Bullying Gestual

Es difícil de identificar, se da

a través de miradas y

ademanes agresivos hacia

el sujeto

Figura 1. Tipos de bullying.

Fuente: Cobo y Tello (2010), Bullying, México: LIMUSA

A partir de las manifestaciones del bullying es posible identificar a los actores. Pero antes es necesario crear la idea de que no solo participa el acosador (bully) y el acosado (bullied), sino hay que tomar en cuenta a todos aquellos que se ven influidos por el fenómeno. A este respecto, Hernández (2013) propone a los siguientes actores: 


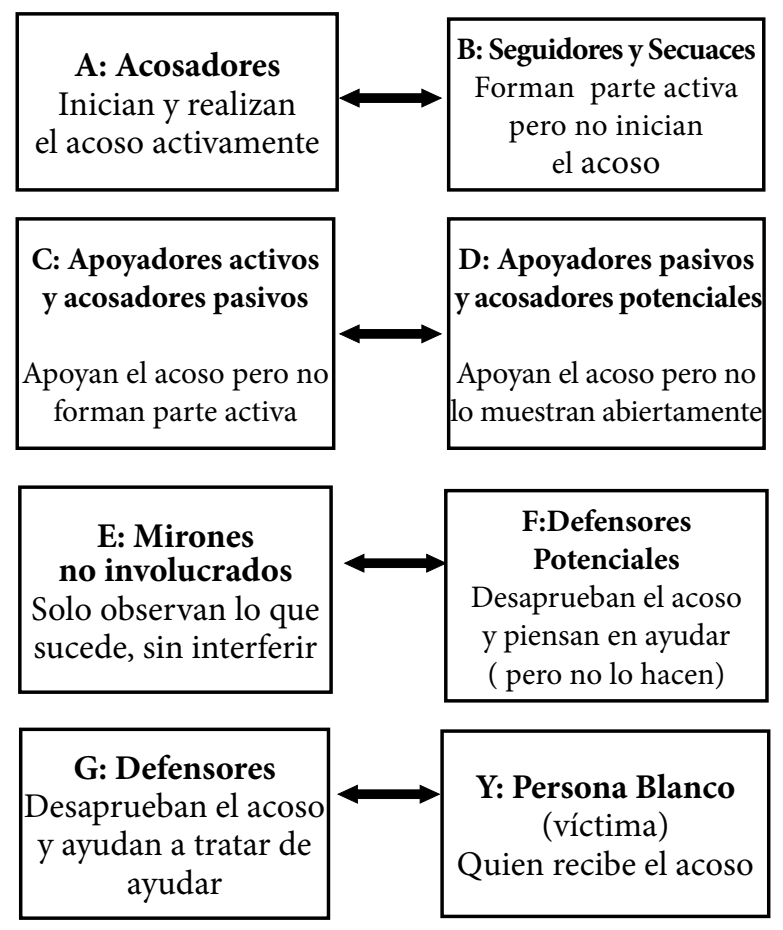

Figura 2. Actores del bulllying.

Fuente: Hernández (2013), Bullying o Acoso Escolar, México.

Con las nociones conceptuales planteadas, es posible visualizar de forma general los elementos importantes de la problemática: ¿Cómo se manifiesta el Bullying en las Escuelas Focalizadas del Municipio de Santa Ana?

El propósito de la investigación fue diagnosticar las manifestaciones del bullying, verificando en el proceso a los actores y el tratamiento disciplinario que se le brinda; asimismo, detectar si el Manual de Convivencia Escolar (instrumento de convivencia dictado por el MINEDUCYT) está actualizado en estas vías. La consecución de lo descrito permitirá elaborar a largo plazo acciones para su paulatina erradicación.

En función de lo anterior, con el estudio se benefició, en primer lugar, a los encargados de
Asistencia Técnica del MINEDUCYT, quienes presiden el programa de escuelas focalizadas del departamento de Santa Ana. En segundo lugar, a las comunidades educativas en estudio, quienes sensibilizaron una realidad latente que, en la mayoría de los casos, por temor social o administrativo es invisibilizada.

\section{Metodología}

Se desarrolló un estudio descriptivo con enfoque investigativo mixto en donde se analizaron las manifestaciones del bullying, teniendo en cuenta sus actores y el tratamiento disciplinario ejercido ante su ocurrencia.

Se investigaron nueve de las treinta escuelas focalizadas, las cuales fueron designadas por los técnicos encargados del programa. Estas instituciones fueron: Centro Escolar Santa Ana California, Complejo Educativo Capitán Gerardo Barrios, Centro Escolar Católico San Lorenzo, Centro Escolar Tomas Medina (El Palmar), Centro Escolar República de Venezuela, Centro Escolar Florinda B. Gonzáles, Centro Escolar Colonia Quiñones, Centro Escolar Juan José Bernal y Centro Escolar Leopoldo Núñez; todas ellas en el municipio de Santa Ana.

En ellas se abordó a 117 sujetos de investigación, entre estos: directivos, docentes y estudiantes; con el propósito de tener las visiones sobre la problemática desde distintas perspectivas.

Se administraron cuestionarios a directivos y docentes partiendo del hecho que, administrativamente, son quienes deben monitorear 
Tabla 1. Población de estudio

\begin{tabular}{|c|c|c|}
\hline $\begin{array}{c}\text { Sujeto } \\
\text { investigado }\end{array}$ & $\begin{array}{c}\text { Cantidad } \\
\text { por escuela } \\
\text { focalizada }\end{array}$ & $\begin{array}{c}\text { Cantidad total } \\
\text { de sujeto }\end{array}$ \\
\hline $\begin{array}{c}\text { Directivos } \\
\text { (Director/Subdirector) }\end{array}$ & 1 & 9 \\
\hline Docentes & 6 & 54 \\
\hline Estudiantes & 6 & 54 \\
\hline Total & $\mathbf{1 1}$ & $\mathbf{1 1 7}$ \\
\hline
\end{tabular}

la problemática. En el caso de los estudiantes, teniendo en cuenta la sensibilidad de la temática y evitando invadir su privacidad, se administró un cuestionario donde se acotaron elementos generales de lo estudiado. Esta variedad de puntos de vista sobre el fenómeno permitió que su descripción fuera objetiva, pues no solo se detectó el lado administrativo, también el humano y real de quienes lo vivencian: los estudiantes.

\section{Resultados}

Se presentan diferentes visiones de una realidad compartida. En algunos casos se visualizarán solo nociones de directivos y docentes, puesto que se necesitaron elementos pedagógicos al respecto.

Para los docentes (91\%) y estudiantes (93\%) dentro de la institución existen manifestaciones de bullying. La realidad puede ser compartida por los dos primeros actores porque ambos interactúan la mayor parte de la jornada escolar en circunstancias donde se facilita el contacto social; y por ende se hacen visibles estas manifestaciones. Por otro lado, el sector directivo (78\%) muestra un comportamien- to porcentual preocupante, pues niegan una realidad que docentes y estudiantes asumen como cierta y activa. Esto puede ser producto de las funciones administrativas que realizan. Pero, de igual manera se denota que existe una carencia de reportes de parte de la comunidad respecto a la problemática.

Al cuestionar sobre acciones específicas de bullying, los estudiantes mencionaron:

- Maltrato entre compañeros frecuentemente (es decir, los mismos sujetos), tales como: empujones, patadas, quecas ${ }^{2}$, aruñones, persecuciones, apodos.

- Juegos bruscos y agresivos en el caso de los niños; y en ocasiones chismes e indirectas de parte de las niñas.

- Desorden en la cafetería, baños y otros lugares muy concurridos en los recreos.

- Risas y gritos cuando alguien comete un error, lo cual es moderado por el docente.

- Lanzamiento de bolas de papel a otros compañeros cuando el docente no los observa.

- Esconder útiles escolares de otros compañeros.

- Agresividad cuando se revisan las tareas. Todos quieren que se les revise al mismo tiempo.

Con esta descripción se observa que los estudiantes tienen dificultad para diferenciar conductas violentas aisladas de un acoso tipo bullying, ya que este se considera como tal en la medida que dura en el tiempo y se

2. Queca: es un modismo empleado por los estudiantes para referirse a golpes en la cabeza. 
le hace a la misma persona. Además, existe un predominio de violencia física y verbal en comparación a una gestual o psicológica.

Los docentes plantean que las manifestaciones se deben a que los estudiantes se ven inmersos a situaciones como:

- Desintegración familiar

- Entorno violento en la comunidad

- Violencia social

- Falta de valores

- Medios de comunicación

- Maras

- Mala comunicación en la escuela y la familia

- Baja autoestima

Aunque son razones válidas, se nota que también no existe una tipificación clara de un estudiante víctima o un agresor, ya que sostienen circunstancias que afectan a todos a nivel social, indistintamente de que sea alguien inmerso en esta problemática.

\section{Actores del bullying}

En este aspecto, se solicitó tanto a directivos y docentes cómo caracterizan a los estudiantes a quienes regularmente se les agrede de diversas formas. Se puntualizaron las siguientes características:

- Tímidos y retraídos

- Introvertidos

- Temerosos de expresarse y muy callados

- Alejados del resto del grupo

- Siempre a la defensiva

- Tristes y agobiados
- Baja autoestima

- Faltan a la escuela con regularidad y no cumplen con sus tareas

- Evitan el contacto grupal y prefieren estar cerca de personas adultas

- Evitan jugar y salir a recreo; y si lo hacen están solos y callados

Asimismo, los estudiantes en el mismo aspecto corroboraron las características anteriores $y$ agregaron que se molesta frecuentemente a aquellos estudiantes que presentan características diferentes como algún problema del habla, físico o de comportamiento.

En contraposición, se indagó la misma caracterización, pero ahora con respecto al estudiante que con frecuencia agrede a otros. En consecuencia, docentes y directivos caracterizaron lo siguiente:

- Prepotentes y ofensivos

- Falsamente seguros de sí mismos

- Violentos

- Irresponsables con sus compromisos académicos

- Intimidantes

- Agresivos

- Extrovertidos

- Superiores en alguna forma a su víctima

- Líderes negativos; poseen seguidores que lo siguen por miedo

- Poseen mal rendimiento académico y expresan con regularidad palabras soeces

- Irrespeto a las reglas y las figuras de autoridad del Centro Escolar

- Destructivos en las pertenencias de los 
demás, incluyendo infraestructura del Centro Escolar.

- Carencia de valores

- Rebeldes y altivos

Los estudiantes coincidieron con lo anterior, pero agregaron que este tipo de estudiante es rechazado por sus conductas violentas; $y$ que a veces los incluyen en su círculo reducido de amigos por el valor social de tener a alguien de ese tipo como aliado para que los pueda defender.

Todos los sectores indagados coincidieron tanto en el agresor como el agredido; pero en el proceso se tuvo dificultad para identificar a otros actores en esta estructura conflictiva, centrándose más en los que dañan o sufren, que los que colateralmente se ven afectados.

\section{Tratamiento disciplinario del bullying}

Los resultados muestran visiones diferentes entre directivos y docentes: por un lado, los directivos plantean que sí existen procedimientos que se orientan a dar tratamiento al bullying (67\%), también sostienen que este instrumento se actualiza con regularidad.

Los docentes, en cambio, sostienen que expresamente no hay procedimientos para atender la problemática (59\%), y que de forma general el proceso a seguir ante conductas de acoso es el siguiente:

1. Llamar la atención e forma discreta al estudiante agresor y confrontarlo, posteriormente, con el agredido para llegar a una conciliación.
2. Si en caso eso no sirve de nada, se recurre al sub-director de la escuela para que él brinde el tratamiento adecuado a la situación (reportes o actividades de beneficio de la escuela). En este caso se levanta una ficha en el expediente del estudiante.

3. Si lo anterior no funciona, se recurre a la Dirección, para que el director contacte al responsable del estudiante y se trate de llegar a un acuerdo conforme a las conductas que este ha mostrado. Se levanta una segunda ficha en el expediente del estudiante.

4. Si el comportamiento no cambia, se elabora una tercera ficha en el expediente del estudiante y se procede a la expulsión de este, ya sea por unos días o en definitiva, dependiendo del caso.

5. En caso se diera un conflicto de carácter legal (violación, asesinato, etc.) se recurre a las instancias necesarias (Consejo Nacional de la Niñez y de la Adolescencia (CONNA), Juzgado Especializado de la Niñez y la Adolescencia (JENA), etc.), obviando todo el proceso anterior, dado su carácter delictivo.

Aunque se manifiesta una estructura disciplinaria con procesos específicos, estos toman cada caso de violencia entre agresor-agredido como un evento independiente; es decir, no existe un seguimiento que denote un registro de regularidad, que permita establecer casos específicos y concretos de bullying. 
Tanto directivos como docentes plantearon que, cuando han acaecido casos de acoso estos se han manifestado en lugares específicos, los cuales son:

- Baños

- Cafeterías

- Canchas

- Atrás de los salones de clase

- Área de la entrada

- Zona de bebederos

- Lugares poco transitados de la escuela

- En los salones de clase cuando estos están vacíos

Este resultado es muy importante, ya que se tienen identificadas las zonas donde las situaciones de violencia entre pares ocurren. Pero la no existencia de procesos específicos en el Manual de Convivencia Escolar en torno a esta problemática dificulta el seguimiento correspondiente.

\section{Apoyo por instituciones externas}

Ambos sectores (directores y docentes) coinciden en que existe apoyo en relación con la convivencia escolar (89\% y 94\%, respectivamente). Específicamente plantearon que este apoyo se manifiesta con el desarrollo de:

- Talleres que sensibilizar al estudiante para que no exista bullying

- Comités y Escuela Inclusiva

- Refuerzo de la autoestima en niños y jóvenes

- Emprendedurismo

- Charlas sobre respeto

- Liderazgo
Ambos sectores plantean que, si bien se les brinda estrategias para trabajar comportamientos violentos de los estudiantes, estas no se apegan a un tratamiento específico del bullying. Eso se traduce, además, en que a nivel disciplinario no existan procedimientos que den el tratamiento y seguimientos adecuados a víctimas de este fenómeno.

Los resultados han mostrado que existe un manejo parcial de la problemática del bullying; que existe conciencia del fenómeno, pero poca claridad para detectar manifestaciones, actores y procedimientos para tratarlo. Inclusive, a nivel directivo este es percibido como un problema administrativo que afecta la imagen de la dirección y subdirección, más que las relaciones sociales de los que convergen en la comunidad educativa.

\section{Discusión}

Partiendo de los resultados obtenidos en la investigación se puede establecer que existen manifestaciones de bullying claras y concretas, con predominancia en las de tipo físico y verbal; además, se identifican características específicas de los agresores y agredidos. En relación al tratamiento disciplinario, no existen procedimientos específicos en el Manual de Convivencia Escolar, pese a que este se ha actualizado con regularidad.

Se puede plantear que existe dificultad para dimensionar y tratar el bullying. A nivel directivo, no se está orientando el trabajo en conjunto con Asistencia Técnico-Pedagógica del MINEDUCYT hacia el fenómeno de 
la problemática. Los directores y subdirectores deben concientizarse que si existe bullying en la escuela no es un problema administrativo, sino un problema contextual que debe ser intervenido para que no fracturen las relaciones de convivencia que convergen en los espacios escolares.

En cuanto a los docentes y los estudiantes se destaca que no existe un manejo de la problemática pues, aunque exista una identificación de situaciones de acoso, no se aprecia una diferenciación entre situaciones de acoso aisladas a las de bullying. Las primeras, aunque dañan, no tienen en muchos casos durabilidad en el tiempo; mientras que las del fenómeno en estudio sí focalizan un comportamiento repitente hacia la misma persona.

Se determinó que la característica de ser una escuela focalizada abre paso a la oportunidad de tener vínculos externos que ayuden al tratamiento del bullying. Pero se detectó que no se está haciendo uso de este recurso. Hacerlo puede llevar a que la comunidad educativa tenga capacitaciones y fortalezca en áreas como la Ley de Protección Integral de la Niñez y Adolescencia (LEPINA), Manual de Convivencia Escolar y bullying.

Una de las medidas que se proponen es la que plantea De Guerrero (2010), en cuanto a la disciplina restaurativa; la cual es una disciplina social que busca mejorar el daño en las relaciones de convivencia desde un enfoque de derechos.

Para Casal (2013), un elemento importante para mitigar el bullying en contextos escola- res es la prevención, mediante la creación de ambientes cooperativos; así como impartir un entrenamiento en resolución de conflictos y mediación escolar que enseñe a los estudiantes a negociar y mediar, y a los docentes y directivos a arbitrar cuando sea necesario.

En respecto a la necesidad actual de las escuelas en estudio, se destaca lo que menciona De Sol (2014), citando a de Olweus (1980), con respecto a un programa de este autor en prevención del bullying. Este consiste en:

1. Evitar intimidar a los demás.

2. Tratar de ayudar a los estudiantes que son intimidados.

3. Incluir a los estudiantes que se quedan fuera de las actividades.

4. Si se sabe que alguien está siendo intimidado, decirle a un adulto en la escuela y a un adulto en casa.

El bullying es un fenómeno que destruye las positivas relaciones sociales que confluyen en los espacios escolares, más no es un problema administrativo; por la tanto, los que administran las escuelas no deben temer reconocer su existencia. Trabajando en equipo y compartiendo responsabilidades se puede intervenir mejor la problemática.

La Asistencia Técnico-Pedagógica del MINEDUCYT es un área de atención directa a escuelas, por lo que no debe dejarse de lado al momento de un proceso de intervención y erradicación del bullying. Hay que incluir a 
todos los actores educativos en la implementación de acciones de intervención, ya que así se logran fortalecer canales de comunicación y el trabajo bajo metas y fines comunes.

Luego de analizar los datos obtenidos y hacer un enfoque holístico del estudio, se pueden plantear las siguientes interrogantes como futuras líneas investigativas al respecto:

- ¿Qué acciones directivas se pueden generar para dar tratamiento específico al bullying en la escuela?

- ¿Cómo se puede involucrar de manera activa a los agentes que colaboran con las escuelas focalizadas en el tratamiento del bullying?
- Conociendo los lugares con mayor vulnerabilidad en los cuales ocurre el bullying dentro de la escuela, ¿pueden generarse estrategias para señalizar y dar atención en esos lugares durante los recreos?

- ¿Qué tipo de disciplina alternativa puede implementarse para dar tratamiento efectivo al bullying?

- ¿Qué acciones específicas puede generar Asistencia Técnico-Pedagógica para orientar de manera asertiva a las escuelas sobre el bullying?

\section{Referencias}

Aguilar, S. (2012). Estrategias Pedagógicas Implementadas para Estudiantes de Educación Media y el Acoso Escolar bullying. San Salvador

Albor-Cohs, G. (2000). BULL-S, Test de Evaluación de la Agresividad entre Escolares, Manual de Referencia. España: Gráficas Ballesteros

Casal, A. (2013). Estudio descriptivo acerca de los efectos del bullying en el rendimiento académico. Cuba: CABA

Cobo, P. y Tello, R. (2010). Bullying. Mexico: LIMUSA

De Guerrero, E. (2010). Disciplina Restaurativa. Santa Ana

De Sol, D. (2014). Prevengamos el Bullying. (pág. 3). Cuba

Díaz, V. (2009). Sindrome bullying en Estudiantes del Ciclo Diversificado del Liceo Bolivariano. Bolivia: Universidad Bolivia

Ministerio de Educación, Ciencia y Tecnología de El Salvador (2013). Plan de Prevención y Seguridad Escolar. Comunicaciones MINEDUCYT, p. 2-3 
Fiabane, F.; Yáñez, N. y Campos, J. (2009). El mejoramiento continuo en las escuelas, articulación entre los establecimientos educacionales y asistencia técnica externa: un estudio de casos exitosos. FONIDE, 3-50

González, J. (2009). "Bullying en El Salvador: un análisis descriptivo en centros escolares de tercer ciclo de educación básica" (Tesis de maestría). Universidad Tecnológica de El Salvador. Recuperado en http://www.psicología-online.com

Hernández, A. (2015). Bullying o Acoso Escolar. México

Moreno, J. (1972). Fundamentos de Sociometría. Buenos Aires: Paidós 